\title{
Direct numerical simulation of the passive heat transfer in a turbulent flow with particle
}

\author{
Marek Jaszczur ${ }^{1, *}$, Marcin Zych $^{2}$, Robert Hanus ${ }^{3}$ \\ ${ }^{1}$ AGH University Science and Technology, Faculty of Energy and Fuels, 30-059 Kraków, Poland \\ ${ }^{2}$ AGH University Science and Technology, Faculty of Geology, 30-059 Kraków, Poland \\ ${ }^{3}$ Rzeszów University of Technology, Faculty of Electrical and Computer Engineering, 35-959 Rzeszów, Poland
}

\begin{abstract}
Turbulent non-isothermal fully developed channel flow with solid particles was investigated through Direct Numerical Simulation combined with the point-particle approach. The focus was on the interactions between discrete and continuous phase and their effect on the velocity and the temperature of the particles. It has been found that low momentum inertia particles have a mean temperature similar to the fluid temperature and this effect is almost independent of particle thermal inertia. For particles with larger momentum, the inertia thermal effect is more complex, particle temperature in the near-wall and buffer region is significantly lower than the fluid temperature. The difference between the fluid mean temperature and particle mean temperature increases along with the momentum response time. This may have important consequences on the chemical reactions, technological processes and on the accuracy of temperature measurement techniques based on seeding particle.
\end{abstract}

\section{Introduction}

Flow with small solid particles or droplets has recently received considerable attention for its relevance to numerous industrial processes, engineering applications and environmental flows. However, researchers have focused mainly on isothermal particle-laden flows. Experiments and numerical computations using Direct Numerical Simulations [1-3] demonstrate that isothermal shear flow has a very complex effect on particle behaviour. Due to particle-turbulence interaction, the distribution of particles and their properties can be highly non-uniform $[4,5]$. This non-uniformity can have very important consequences on the process-for example, affecting the efficiency of the chemical reaction or the properties of the mixtures.

A great deal of work in this field has been done towards understanding the influence of particle inertia on dispersion phenomena [6]. Even though we now have a better understanding of turbulence modification by small solid particles, the mechanisms are complex and not easy to predict [7].

In understanding particle-turbulence interactions, Direct Numerical Simulation (DNS) and Large Eddy Simulation (LES) plays an important role [8-10]. In the case of flows laden with a large number of small particles, Eulerian-Lagrangian point-particle DNS has proven quite successful in studies of particle-turbulence dynamics. Several studies have focused on the particleturbulence interaction in homogeneous isotropic turbulence by two-way coupling [11]. They found that increasing the mass loading of small solid particles reduces turbulent kinetic energy. In this research, the preferential concentration of light particles led to an increase in the small-scale velocity fluctuation. The effect of the two-way coupling increases the rate of the kinetic energy of the turbulence transfer from the large scale towards the small scale. This may result in a larger dissipation rate [12].

An increasing amount of research has recently been reported for fully developed particle-laden turbulent channel flow. The application of DNS to the study of particle deposition in boundary layers by Wang and Squires [13] has clearly shown accumulation of heavy particles in the low-velocity streaks.

While there are a large number of applications from this including combustion, spray drying, and heat exchanger, very small attention has been paid to particle analysis in the non-isothermal flow [14-16]. This type of flow is known to be affected by the thermal layer through various mechanisms [17-19]. The modulation of the turbulent heat transfer due to particle-fluid interactions can be important at even low loadings [20,21]. The total heat capacity of the gas-particle mixture may be modified and because of that turbulent heat transfer modulation [22] may be the next important effect. The particle-wall and inter-particle collisions are the next effects expected to play a significant role [23]. The collision effect even in the flow with a very low volume fraction can seriously affect the dynamics of the two-phase flow and the heat transfer.But still today only few research has concentrated on the particle-laden flow in non-isothermal cases and the concentration is more on isolated effects than on overall particle-turbulence interaction [24].

*Corresponding author: marek.jaszczur@agh.edu.pl 
Presented work thoroughly investigates the dynamics of small heavy inertial particles in non-isothermal turbulent fully developed channel flow. The cases considered are representative of a large family of phenomena known in the literature as multiphase flows, which contain both a continuous phase and a dispersed phase.

Fully-developed particle-laden non-isothermal turbulent channel flow [25] is investigated using Direct Numerical Simulation (DNS) combined with the Lagrangian particle tracking technique, which is used to track a large number of individual particles, using the point-particle approach.

In the configuration presented here, small solid dense particles carried by the fluid forces are influenced by the turbulent flow. The simulations are performed without gravity at shear Reynolds number $\mathrm{Re}_{\tau}=150$ and for a molecular Prandtl number $\operatorname{Pr}=1.0$. The effect of the particle diameter and density on the statistical quantities is considered. The key issue is to examine, on the basis of statistics, particle dispersion with reference to the particle hydrodynamic and thermal relaxation times.

\section{Mathematical model and numerical methods}

For computation of wall-bounded turbulent flow with particle driven by the uniform pressure gradient between the Eulerian-Lagrangian point-particle approach has been used. The continuous-phase is solved using DNS techniques for incompressible flow which is combined with the tracking of the individual particles. In the simulation, the transfer of momentum between the fluid and the point-particle is computed through a force located at the geometrical centre of the particle and is determined base on the velocities of the particle and of the surrounding fluid. The temperature is determined base on velocities of the particles and fluid and the temperatures of the particle and of the surrounding fluid. This approach is valid if the particles are significantly smaller than the smallest flow scales both for velocity and temperature. The flow in the channel (Fig.1.) is assumed to be fully developed both thermally and hydrodynamic.

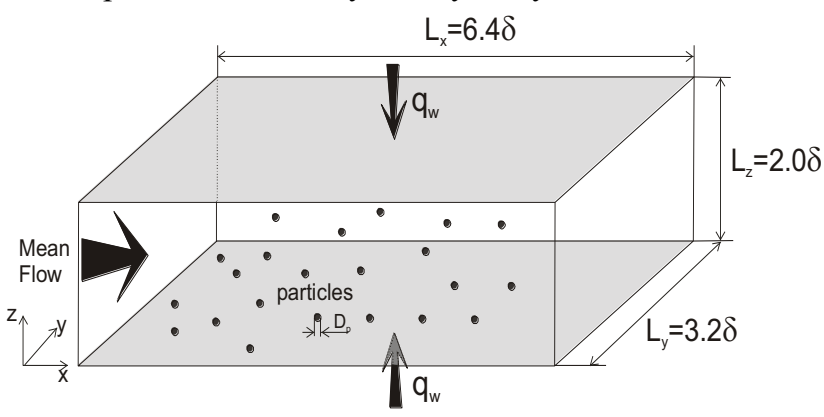

Fig.1. Flow geometry.

The streamwise wall-normal and spanwise coordinate are denoted x,y,z. The wall heat flux $q_{\mathrm{w}}$ is assumed to be uniformly distributed on walls and the working fluid is assumed to be Newtonian fluid with constant properties.
Temperature is treated as a passive scalar. Under this assumption, the continuity, Navier-Stokes and energy equations are described, as follows:

$$
\begin{gathered}
\nabla \cdot \vec{U}=0 \\
\frac{\partial \vec{U}}{\partial t}+(\nabla \vec{U}) \cdot \vec{U}=-\frac{1}{\rho} \nabla P+\nu \nabla^{2} \vec{U} \\
\frac{\partial T}{\partial t}=-\nabla(\vec{U} \cdot T)+\alpha \nabla^{2} T
\end{gathered}
$$

For small heavy particles with diameter smaller than Komogorov scale in the equation of particle motion the only significant force is the drag and the governing equations can be written as:

$$
\begin{aligned}
& \frac{d \vec{v}}{d t}=C_{d} \frac{R e_{p}}{24} \frac{1}{\tau_{V}}(\vec{u}-\vec{v}) \\
& \frac{d T_{p}}{d t}=\frac{N u}{2} \frac{1}{\tau_{T}}\left(T-T_{p}\right)
\end{aligned}
$$

Where $\vec{u}$ is the velocity of the surrounding fluid interpolated at the centre of the particle and $\vec{v}$ is the velocity of the particle. The particle Reynolds number, $\mathrm{Re}_{\mathrm{p}}$, and the hydrodynamic particle-relaxation-time $\tau_{V}$, are defined as:

$$
R e_{p}=\frac{|(\vec{u}-\vec{v})| D_{p}}{v}, \tau_{V}=\frac{\rho_{p}}{\rho} \frac{D_{p}^{2}}{18 v}, C_{d}=\frac{24}{R e_{p}}
$$

where $\rho_{p}$ and $\rho$ are the particle and fluid densities, $D_{p}$ is the diameter of the particles and $C_{d}$ is the drag coefficient for Stokes flow. The volume fraction of particles is assumed to be small enough such that particle-particle interactions are negligible. The equation for the temperature of particles, assuming small Biot number and thermal particle-relaxation-time, $\tau_{T}$, can be written in the form:

$$
\tau_{T}=\frac{\rho_{p} c_{p} D_{p}^{2}}{12 k}, \operatorname{Pr}=\frac{v \rho c}{k}
$$

where $T$ is the temperature of the surrounding fluid interpolated at the centre of the particle and $T_{p}$ is the temperature of the particle and $\tau_{T}$ is the thermal particlerelaxation-time. In order to take into account effect of particles relative velocity and fluid number dependence on the Nusselt number the value of the Nusselt is calculated from the Ranz-Marshall correlation:

$$
N u=2+0.6 \operatorname{Re}_{p}^{1 / 2} \operatorname{Pr}^{1 / 3}
$$

where the Pr is the Prandtl number.

The position, flow, and particle quantities are normalised by the channel half-width, $\delta$ the friction velocity, $u_{\tau}$ and the friction temperature, $T_{\tau}=q_{w} /\left(\rho \cdot \mathrm{c}_{\mathrm{p}} \cdot \mathrm{u}_{\tau}\right)$. The governing equations (1)-(3) are solved numerically using the fractional step method on a staggered grid. Adams-Bashforth method was used for time advancement of all terms with the step determined by the Courant criterion. The Poisson equations for pressure is solved using Fast Fourier Solver. The equation of the particle motion (4) and the equation of the particle temperature (5) are solved use an Adams-Bashforth scheme for the 
time-advancement and a tri-linear interpolation for the velocity and temperature field at the centre of the particle. To force the fluid motion, a constant pressure gradient is imposed along the streamwise direction. Periodic boundary conditions are imposed in both streamwise and spanwise directions for the hydro and thermal fields. In order to apply periodicity in the streamwise direction, the following transformation of the pressure and temperature is made:

$$
p_{i}=p-\left|\frac{d\langle\bar{p}\rangle}{d x}\right| x \quad, \quad T_{i}=T-\left|\frac{d\langle\bar{T}\rangle}{d x}\right| x
$$

where

$$
\left|\frac{d\langle\bar{p}\rangle}{d x}\right|=-2 \rho u_{\tau}^{2} / \delta \quad, \quad\left|\frac{d\langle\bar{T}\rangle}{d x}\right|=\frac{q_{w}}{\rho c_{p} U_{b} \delta}
$$

\section{Numerical Results}

The numerical results of the most relevant statistics for non-isothermal particle-laden flow are presented and analysed. Fluid and particle statistics are presented at a statistically steady state at Reynolds number $\operatorname{Re}_{\tau}=150$ and for Prandtl number $\operatorname{Pr}=1.0$. Particle statistics for particle presented in Table 1 are computed assuming considered variable over particles in a certain test volume, constituted by wall parallel fluid slabs and by averaging the number of particles in the sampling volume.

Table 1. The particles and grid data.

\begin{tabular}{|c|c|c|c|}
\hline$d_{p}^{+}$ & $\mathrm{St}=\tau_{p}^{+}$ & $\mathrm{St}_{\mathrm{T}}=\tau_{T}^{+}$ & $N_{x} \times N_{y} \times N_{z}$ \\
\hline 0.15 & 1 & $0.1 / 1.0 / 10.0$ & $128 \times 128 \times 64$ \\
\hline 0.15 & 1 & $0.1 / 1.0 / 10.0$ & $256 \times 256 \times 256$ \\
\hline 0.342 & 5 & $0.5 / 5.0 / 50.0$ & $128 \times 128 \times 64$ \\
\hline 0.765 & 25 & $2.5 / 25.0 / 250$ & $128 \times 128 \times 64$ \\
\hline
\end{tabular}

It is much more time-consuming to get a statistically steady state for particle than for fluid. For the same Reynolds number and different Stokes number, the developed times are very different. For small particles (small St number), the time is shorter while for intermediate particles it is enormously long. In the Fig.2, the time evolution of the particle concentration in the near-wall region $\left(z^{+}=1.0\right)$ has been presented up to $t^{+}=30000$. Particle field for $\mathrm{St}=1$ was used as an initial condition to generate these profiles; for this value, particle immediately achieves a steady state. An increase or decrease in particle concentration in the near-wall region can be observed for other Stokes numbers.

Obviously, for the $\mathrm{St}=5$ it takes significantly longer to reach a steady concentration at this location than for the $\mathrm{St}=1$ where maximum concentration $C$ is about 2.5. This is consistent with the results found by other researchers and shows that the maximum particle deposition rate occurs for the particle $\mathrm{St}=25$. Then, as the relaxation time increases, particle concentration decreases. It was found by Elghobashi and Truesdell [26] that at $\mathrm{Re}_{\tau}=150$ a peak of concentration occurs for $\mathrm{St}=25$. The particle with this Stokes number is the most responsive to the turbulence structures in the near wall region.

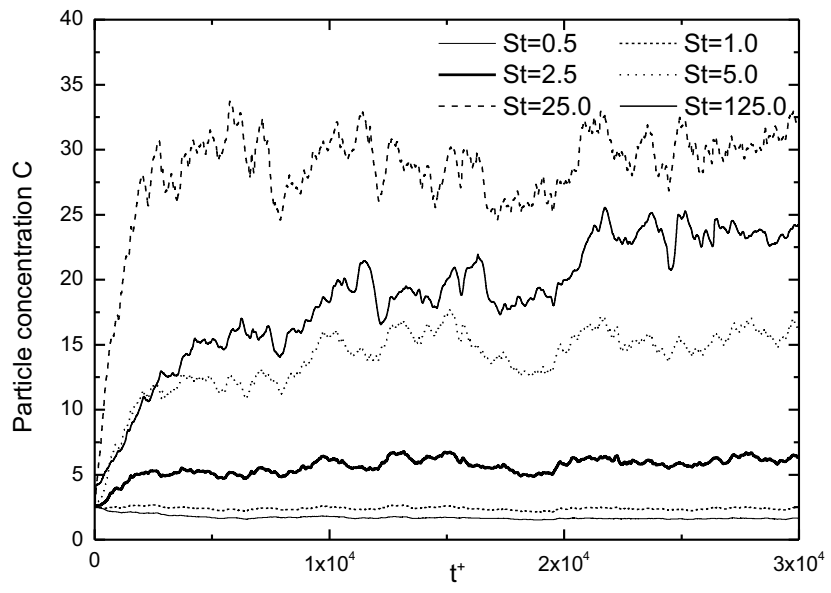

Fig. 2. Particle concentration evolution at the near-wall region $\left(\mathrm{z}^{+}=1.0\right)$ as a function of non-dimensional time $t^{+}$.

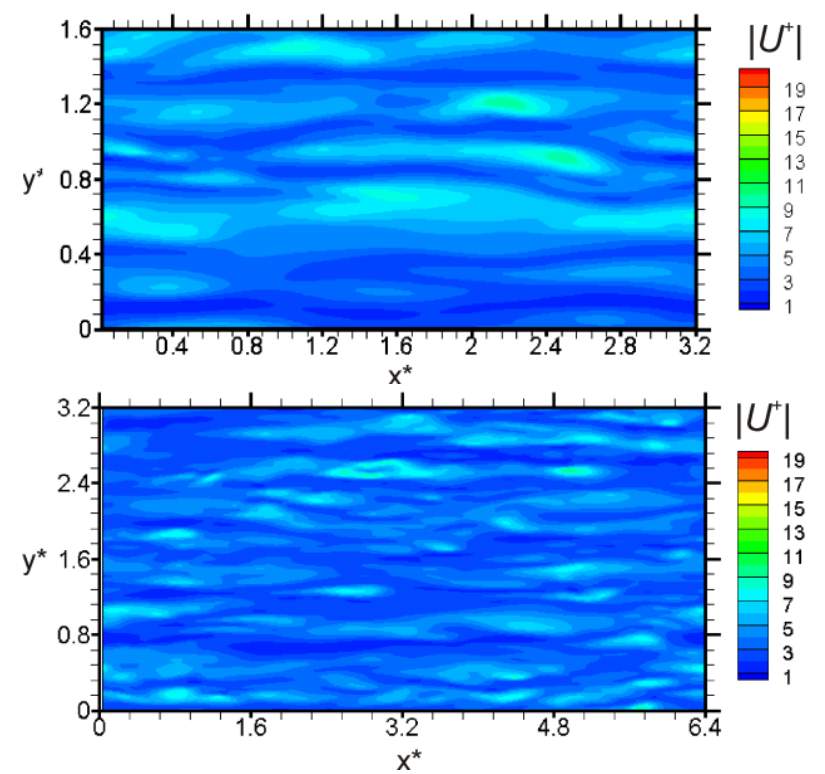

Fig. 3. Instantaneous velocity with different grid resolution: coarse(top), fine (bottom) in XY cross-section at $z^{+}=3.6$ for $\mathrm{Re}_{\tau}=150, \operatorname{Pr}=1$.

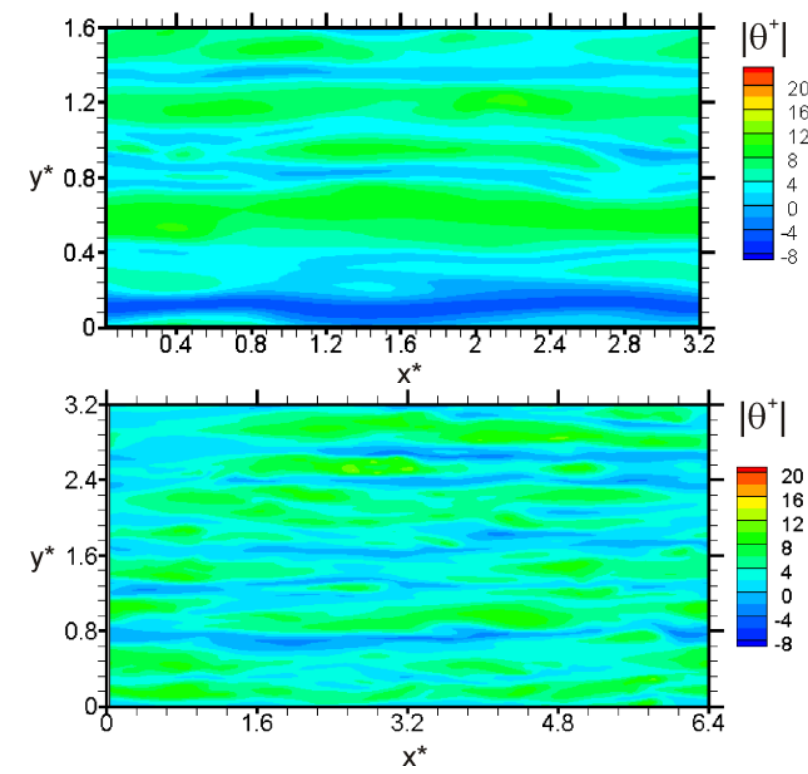

Fig. 4. Instantaneous temperature with different grid resolution: coarse(top), fine (bottom) in XY cross-section at $z^{+}=3.6$ for $\mathrm{Re}_{\tau}=150, \operatorname{Pr}=1$. 


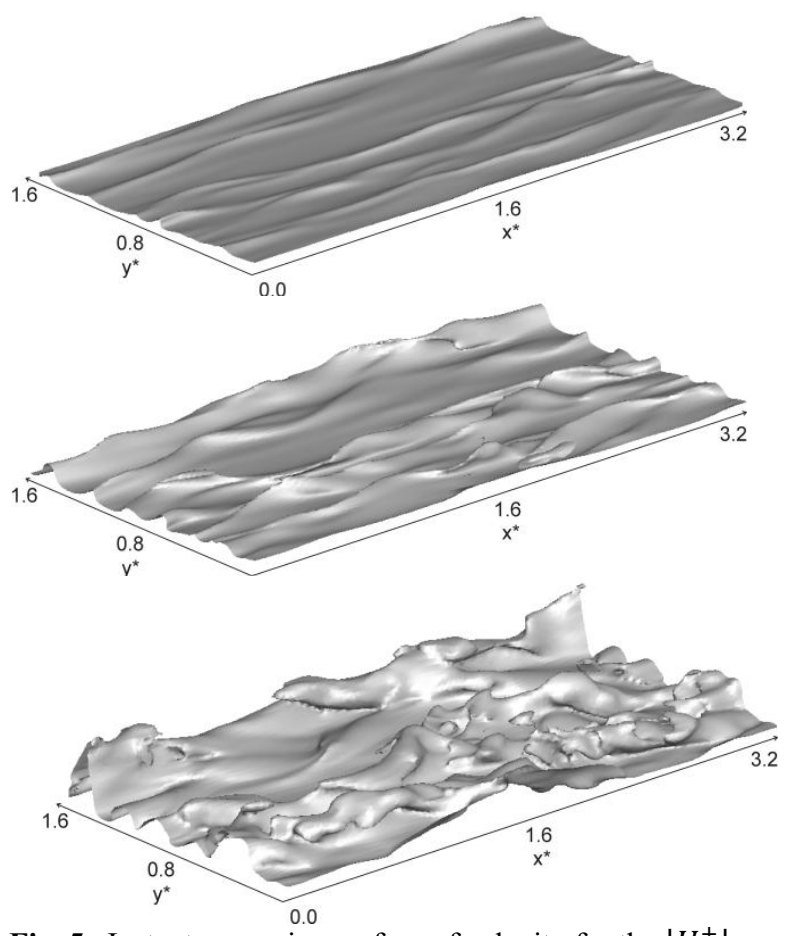

Fig. 5. Instantaneous iso-surface of velocity for the $\left|U^{+}\right|=$ $\theta^{+}=3.6 ; 10.0$ and 15.0 (top, middle, bottom).

Fig. 3 and Fig. 4 show the effect of the grid resolution and the domain size, which was increased in streamwise and spanwise directions on the velocity magnitude. Two grid resolutions were tested at Reynolds number $\operatorname{Re}_{\tau}=150$ : (coarse) $N_{x} \times N_{y} \times N_{z}-128 \times 128 \times 64$ with the domain $L_{x} \times L_{y} \times L_{z}$ equal to $2 \pi \delta \times 1 \pi \delta \times 2 \delta$; (fine) $256 \times 256 \times 256$ and domain equal $4 \pi \delta \times 2 \pi \delta \times 2 \delta$. Each plot was obtained in different DNS computations and it is not possible to show simultaneously differently resolved fields. To show the flow and thermal field complexity, the iso-surface of the constant velocity magnitude $\left|U^{+}\right|$ and temperature $\theta^{+}$are presented in Fig. 5 and Fig.6. for the bottom half of the channel. The iso-surface values for both properties are the same and are equal to 3.6, 10.0 and 15.0. Particularly the $\left|U^{+}\right|=\theta^{+}=3.6$ represents on average the mean temperature and mean velocity at position $z^{+}=3.6$. It can be seen that instantaneous fields have complex surfaces which reflect the influence of the large flow structures described previously. Flow and thermal fields look like valleys with peaks and troughs.

For the magnitude of velocity, the minimum instantaneous value is exactly zero (and obviously the mean value and its fluctuation at the wall are zero) which is a consequence of non-slip boundary conditions; however, the instantaneous temperature $\theta^{+}$at the wall is not necessarily zero. In the introduced normalisation system, the mean value at the wall is zero but the instantaneous value can reach a much lower value. Temperature fluctuation at the wall is also non-zero (boundary condition - heat flux at the wall). This obvious difference causes "empty spaces" on the temperature isosurface.
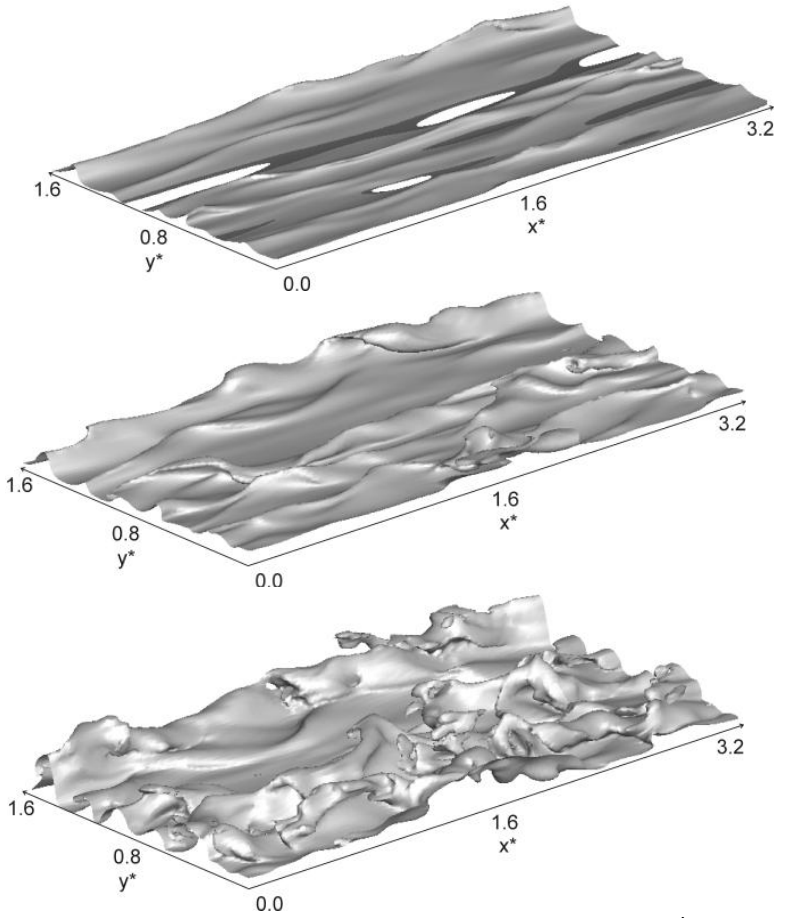

Fig. 6. Instantaneous iso-surface of temperature for the $\left|U^{+}\right|=$ $\theta^{+}=3.6 ; 10.0$ and 15.0 (top, middle, bottom).

Increasing the level of the isosurface up to $\left|U^{+}\right|=\theta^{+}$ $=10.0$ does not cause the valley structures to become dissimilar, but the peaks are higher. The distance between the peaks here is similar to the distance between the peaks for iso-surface 3.6. The mean value for velocity magnitude and temperature equal to 10.0 is most representative for the location $z^{+} \approx 11$. The last isosurface presented here is plotted for 15.0. It can now be seen that the iso-surface for the temperature and velocity magnitude is very complex and in same areas the surface "starts to detach" from the main surface.

Obtaining particularly a statistically steady state for particle position is very difficult and required much more time than for any other development. From Fig.7 it can be seen that at the lowest Stokes number $(\mathrm{St}=1)$ the particles are nearly uniformly distributed in the near wall region and are not far from a random distribution in the center region. There are a few empty spots - places with a low number or no particles.

With increasing Stokes number up to $\mathrm{St}=5$, more particles can be seen in the near-wall region. At the same time, a significantly smaller number of particles is observed in the centerline region, and with a distribution different from the other cases. $\mathrm{St}=5$ particles in the center region are far from any uniformity and form structures at a very high concentration while at the same time leaving many areas entirely devoid of the 1.5 million particles. Further increases in the Stokes number up to $\mathrm{St}=25$ show a more pronounced effect of the increasing concentration near the wall and many more particles are accumulated close to the wall. Additionally, in the near-wall region the main particle accumulations occur in nearly straight streaks elongated in the streamwise direction. 

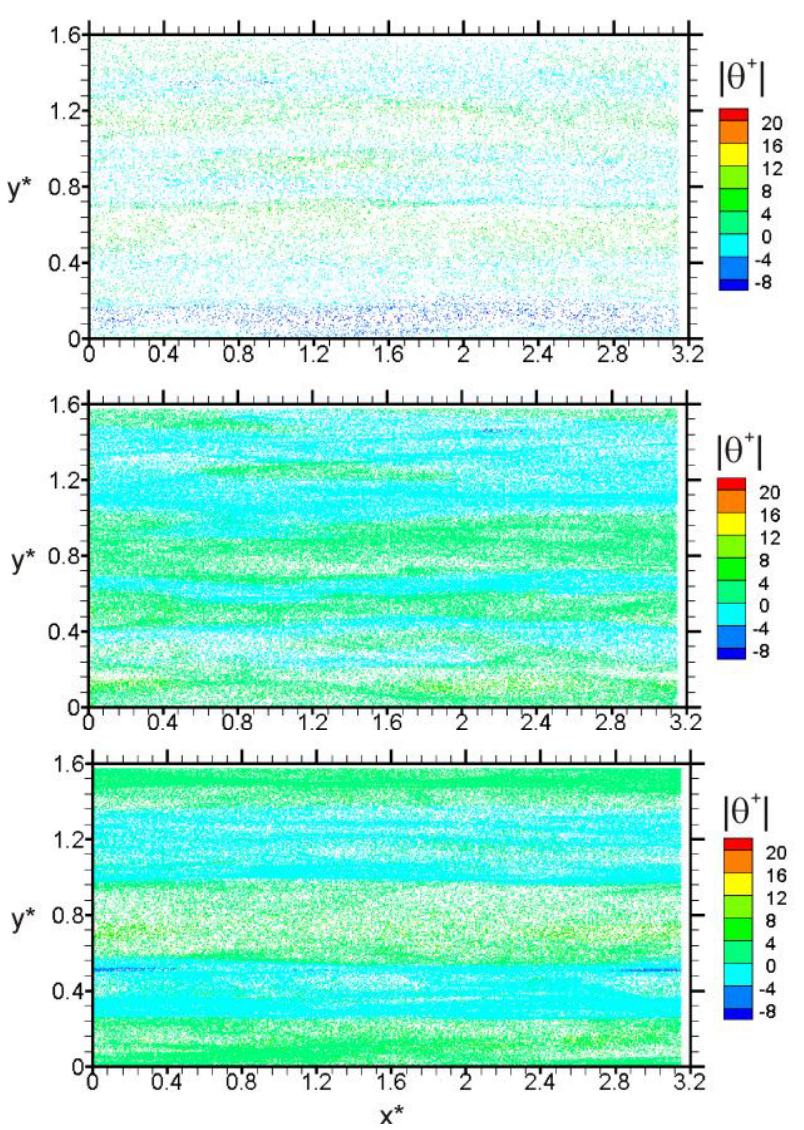

Fig. 7. Instantaneous particle field colored by temperature at $\mathrm{z}^{+}=3.6$ for Stokes number $\mathrm{St}=\mathrm{St}_{\mathrm{T}}=1,5,25$ (top, middle, bottom).

Fig. 8 presents profiles of the mean streamwise velocity and temperature for the fluid and particles nondimensionalized by the wall shear velocity. The results show a modification of the mean particle dynamics with increasing particle.

For all of the momentum Stokes numbers considered here $(\mathrm{St}=1,5,25)$, the mean streamwise velocity lags the fluid velocity except for in the channel centre region. This lag is larger for particles with larger inertia. In fully developed channel flow independent of the particles relaxation time for long enough statistics to be determined, velocity (and temperature) could be expected to be identical for the fluid and particles.

For the $\mathrm{St}=1$ particles with a response time equal to the fluid response time, the difference between fluid and particle velocity is very small. For 25 particles the difference became large and the particles' average velocity could become $20 \%$ lower than the fluid velocity. The difference between the fluid temperature and particle temperature for heavier particles is much larger than observed for the fluid.

In Fig.9 particle, velocity fluctuation for the streamwise, spanwise and wall-normal components are plotted as a function of the wall-normal coordinates in wall units. The results are presented for $\mathrm{St}=1,5,25$. including the fluctuation of the fluid velocity components. For the streamwise component, the observed effect is the opposite of that for the particle mean streamwise velocity. The velocity fluctuation increases along with the Stokes number. Even for the lightest particles considered here the difference in fluid and particle fluctuation is large.
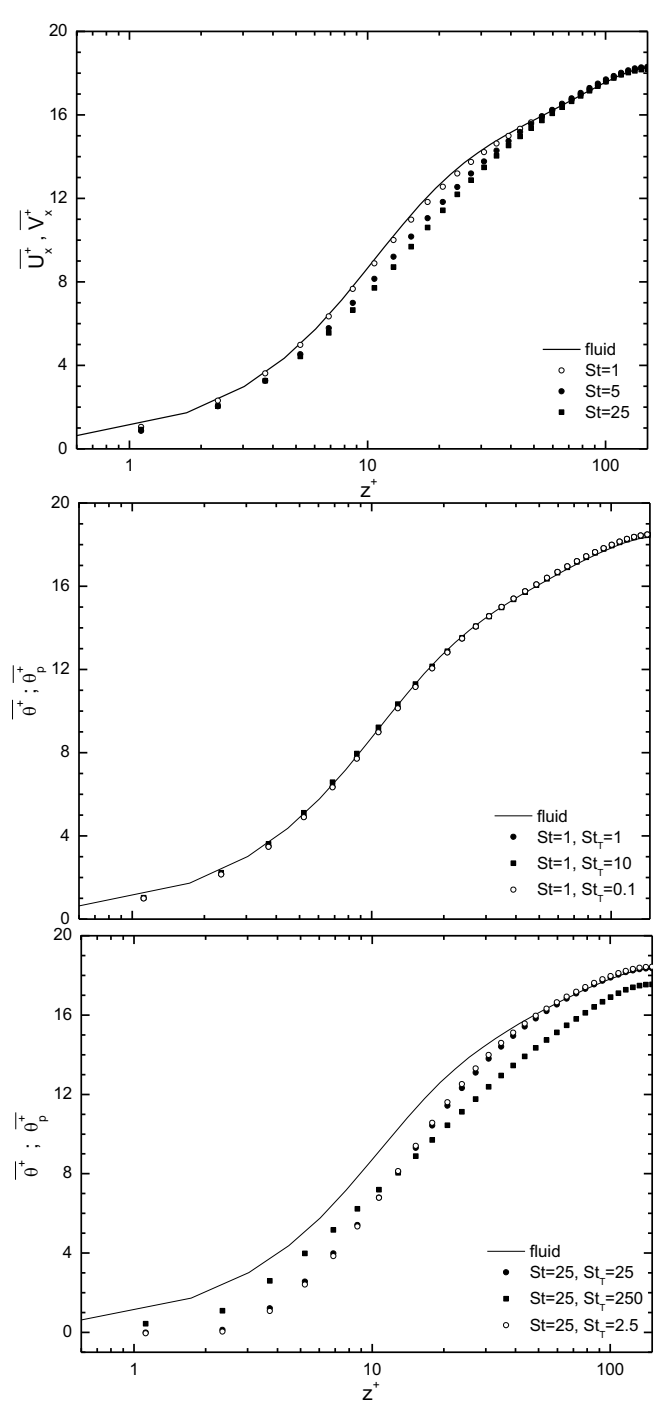

Fig. 8. Mean streamwise velocity profile (top) and temperature (middle, bottom) for the fluid (solid line) and for particles.
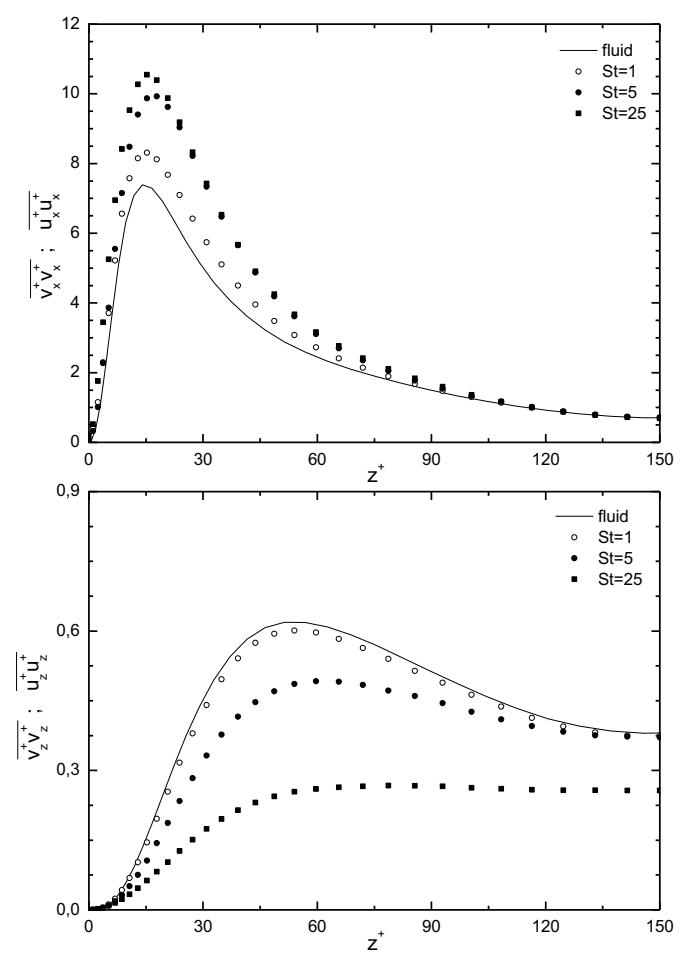

Fig. 9. Mean particle and fluid second order statistics for the $\mathrm{St}=1,5$, and 25 particles and for the fluid $\mathrm{Re}_{\tau}=150$. 


\section{Conclusions}

The DNS of solid particles without gravity was performed with the aim of extending knowledge about turbulence particle interaction in the non-isothermal flow.

The results presented in this paper for continuous phase show that the streaks are complicated but the spatial distance between them and the length is roughly constant and the thermal streaks are less elongated. The spatial distance in the near-wall region in the spanwise direction is about $L_{y}=100$, while the streamwise distance $L_{\mathrm{x}}$ is about 10 times larger.

One and half million of particles introduced in the fully developed flow for each Reynolds number have been considered in this paper. Particles were characterised using particle relaxation time for momentum and for the thermal response, which is proportional to the Stokes numbers $\mathrm{St}$ and $\mathrm{St}_{\mathrm{T}}$. Particle inertia can be considered as a low-pass filter that strongly influences particle response to the turbulence, particularly to large-scale structures.

The results show that temperature and velocity, as well as concentration, are strongly influenced by the Stokes number with respect to the Reynolds number. In the near wall region, the particle is transported mainly by the large turbulent coherent structures and strongly depends on the ejections and sweeps. But because of particle inertia, the particle re-entrainment process is less promoted than deposition in the viscous region. Particle segregation occurs in particular places and has obtained what is call known in the literature as a preferential concentration. This mechanism influences not only particle position and, finally, particle distribution, but also influences particle temperature. Due to preferential concentration, a similar effect causes what can be called preferential temperature. Strong coherent structures segregate particle very effectively and by doing so also exert an influence on the particle temperature distribution. Preferential concentration occurs over a wide range of flows of many practical applications.

The mean temperature for the fluid in the boundary layer region is higher than the mean temperature for the particles. A similar effect can be found for the mean streamwise velocity, but the difference for the temperature is larger. In the centre region of the channel the particles, regardless of the thermal response time, are almost symmetrically distributed around the mean. Particles in this region form highly concentrated bands that influence the distribution, which became narrow and much higher. The difference between differently thermally responsive particles can also be very large. This may have important consequences on the chemical reactions, technological processes and on the accuracy of temperature measurement techniques based on seeding particle.

\section{Acknowledgements}

The present work was supported by the Polish Ministry of Science (Grant AGH No. 11.11.210.312).

\section{References}

1. R. D. Moser, J. Kim, N. N. Mansour, Phys. of Fluids. 11, 943-945 (1999)

2. J. Kim, P. Moin, Turbulent Shear Flows 6, 85-96 (1989)

3. N. Kasagi, Int. J. of Heat and Fluid Flow 19, 125-134 (1998)

4. J.McLaughlin, Phys. Fluid A1, 1211-1224 (1998)

5. M. Rashidi, G. Hetsroni, S. Banerjee, Int. J. of Multiphase Flow 16, 935-949 (1990)

6. J. G.M. Kuerten, C. W. M. van der Geld, B.J. Geurts, Phys. of Fluids 23, 123301-123309 (2011)

7. N. Anbuchezhian, K. Srinivasan, K. Chandrasekaran, R. Kandasamy, Applied Mathematics and Mechanics 33, 765-780 (2012)

8. L.M. Portela, R.V.A. Oliemans, Int. J. Numer. Fluids 43, 1045-1065 (2003)

9. D. Rouson, J. Eaton, Proc. of $7^{\text {th }}$ Workshop on TwoPhase flow Predictions, Erlangen (1994)

10. B. Lessani, M.H. Nakhaei, Int. J. of Heat and Mass Transfer 67, 974-983 (2013)

11. L. Wang, M. Maxey, J. Fluid Mech. 256, 27 (1993)

12. C.D. Dritselis, N.S.Vlachos, Int. J. of Multiphase Flow 37, 706-721 (2011)

13. Q.Wang, D.Squires, J. of Multiphase flow 22, $667-$ 683 (2001)

14. N. Kasagi, Y. Tomita, A. Kuroda, Journal of Heat Transfer 114, 598-606 (1992)

15. N. Kasagi, Y. Ohtsubo, Turbulent Shear Flows 8, 97$119(1993)$

16. H. Kawamura, H. Abe, Y. Matsuo, Int. J. of Heat and Fluid Flow 20, 196-207 (1999)

17. H. Kawamura, H. Abe, K. Shingai, Proceedings of the $3^{\text {rd }}$ Int. Symp. on Turbulence, Heat and Mass Transfer, Japan (2000)

18. Y. Na, D. V. Papavassiliou, T. J. Hanratty, Int. J. of Heat and Fluid Flow 20, 187-195 (1999)

19. L. Redjem-Saad, M. Ould-Rouiss, G. Lauriat, Int. J. of Heat and Fluid Flow 28, 847-861 (2007)

20. M. Jaszczur, Archives of Mechanics 63, 77-91 (2011)

21. M. Jaszczur, Direct and Large-Eddy Simulation VIII, Springer-Verlag, Berlin (2011)

22. F. Zonta, C. Marchioli, A. Soldati, Acta Mechanica 195, 305-326 (2008)

23. Y.H. Dong, L.F. Chen, Int. J. of Heat and Mass Transfer 54, 1168-1178 (2011)

24. I. Tiselj, E. Pogrebnyak, C. Li, A. Mosyak, G. Hetsroni, Physics of Fluids 13, 1028-1039 (2001)

25. M. Ould-Rouiss, L. Redjem-Saad, G. Lauriat, A. Mazouz, Int. Comm. in Heat and Mass Transfer 37, 958-963 (2010)

26. S. E. Elghobashi, G.C. Truesdell, Physics of Fluids, A5, 1790-1801 (1993) 